\title{
Sentinel Node Biopsy After Neoadjuvant Systemic Therapy for Breast Cancer: The Method Matters
}

\author{
Harry D. Bear, MD, PhD, and Kandace P. McGuire, MD \\ Division of Surgical Oncology, Massey Cancer Center, Virginia Commonwealth University, VCU Health, Richmond, VA
}

The transition from axillary lymph node dissection (ALND) to sentinel lymph node biopsy (SLNB) has been a major advance over the past 30 years, leading to decreased morbidity of breast cancer treatment. ${ }^{1,2}$ Similarly, demonstrations that neoadjuvant systemic therapy (NST) is equivalent to adjuvant therapy have paved the way to deescalation of local and regional therapies, despite some concerns about possible increased risk of local recurrence in the breast. ${ }^{3-8}$ For example, now NST often is used to avoid total mastectomy, to reduce the magnitude of partial mastectomies and potentially to avoid ALND and/or regional nodal irradiation, even in patients with clinically positive nodes at the time of diagnosis. ${ }^{6,9,10}$ The use of SLNB in place of ALND after NST has been widely accepted for patients with clinically negative nodes at presentation, but the same approach has been more controversial for patients with clinically and pathologically proven positive nodes. Current NCCN guidelines regarding surgical lymph node staging after NST state that if axillary node imaging or needle biopsy is negative, then SLN should preferably be performed after NST. ${ }^{11}$ It also notes that NST "may allow SLNB alone if a positive axilla is cleared with therapy." ASCO guidelines from 2016 suggest that SLNB may be offered to patients who receive NST but suggest caution for patients with large or bulky metastatic axillary nodes. ${ }^{12}$ Conversion of positive to negative nodes is more likely to occur with triple negative or HER-2 ${ }^{+}$breast cancers, and multiple studies have

(C) Society of Surgical Oncology 2019

First Received: 9 March 2019;

Published Online: 24 April 2019

H. D. Bear, MD, PhD

e-mail: hdbear@vcu.edu shown that neoadjuvant chemotherapy results in a fairly consistent conversion rate from clinically positive nodes to pathologically negative nodes in the range of $40 \% .^{10,13-17}$

The main concerns regarding the use of SLN for patients who present with positive nodes and then receive NST have been whether the identification rate is adequate and whether the false-negative rate (FNR) is sufficiently low. The failure to map is not as big an issue as the FNR, as the fallback position from failure to identify a SLN would be to proceed with ALND in patients with positive nodes prior to NST. False-negative SLN (i.e., histologically negative SLN despite residual cancer in other axillary nodes), on the other hand, has significant potential consequences. These include misrepresentation of the prognosis for these patients, especially for TNBC and HER- $2^{+}$cancers, for whom the absence of a pathologic complete response, especially residual cancer in lymph nodes, signifies a higher likelihood of subsequent recurrence and death. ${ }^{3,18,19}$ Residual disease in axillary nodes has become even more significant since recent reports, such as the CREATE-X and Katherine trials, have shown that adjuvant treatment for patients with residual cancer after NST reduces the risk of recurrence for TNBC or HER-2 $2^{+}$cancers, respectively. ${ }^{20,21}$ Similarly, conversion of positive nodes to negative may be a basis for omitting regional nodal irradiation, depending on the results of trials like NSABP B-51. ${ }^{22}$

Previous studies of the identification rates and accuracy of SLN biopsy, including meta-analyses, have been interpreted in varying ways. Multiple retrospective studies and meta-analyses of SLN biopsy performed with ALND have been consistent in finding FNR in the range of $7-13 \% .{ }^{13,23-26}$ The prospective Z1071 trial found that the false-negative rate overall for patients with clinical N1 disease and two or more SLN found was $12 \%$, a bit higher than the target rate of $10 \% .{ }^{14}$ Patients with clinical N2 nodal disease (fixed or matted nodes) were included in the 
study but were not analyzed as part of the primary endpoint. A number of controllable aspects of the method were associated with lower FNR, including using dual tracer techniques (radioactive and color) and confirmation of removal of the initially biopsied positive node by placing a clip and confirming retrieval of the clipped node at the time of the SLNB. More recently, techniques for marking the needle biopsy-positive nodes and verification that they are removed, combined with SLN mapping and removal, can reduce the FNR to as low as $2 \% .{ }^{27-31}$ These methods have included placing a clip in the node at the time of biopsy, identification of the clip by intraoperative ultrasound and specimen radiograph, ${ }^{125} \mathrm{I}$ seeds placed at the time of biopsy or before surgery, or tattooing positive nodes with India ink. ${ }^{27,28,32}$ Nonradioactive marker options, such as using magnetic, radiofrequency, or radar technology markers, are additional options. The importance of this is highlighted by the fact that $20 \%$ or more of marked positive nodes are not among the nodes identified by mapping, even with use of dual tracers..$^{27,30,31,33}$ For us, this means that SLN is generally reserved for patients with no more than two to three abnormal nodes on imaging, because biopsy and marking more than this is not generally feasible. A more recent study suggests that ALND is actually associated with improved survival in patients presenting with N2-3 disease. ${ }^{16}$

In this issue of the Annals of Surgical Oncology, SoYoun et al. reported a small, randomized trial of SLN mapping after NST with radioisotope (RI) alone versus a dual method (DM) with radioactive tracer plus a fluorescent dye, indocyanine green (ICGF) ${ }^{34}$ They found that the rate of SLN identification was equal in both groups (93.8\% with RI alone vs. $98.3 \%$ with DM), and they claim that the identification rate with ICGF alone "exceeded" that for RI alone (94.7 vs. 93.8\%). Neither of these differences are statistically or clinically significant, and the sample size was predicated on the assumption that the identification rate with RI alone would be only $80 \%$. Both rates are similar to the identification rate in the Z1071 trial (92.7\%, including the N2 patients). Although a second SLN was found more often with ICGF than with RI, this was based on only 30 patients and was not statistically significant. Because they did not actually test ICGF alone versus RI alone, their conclusion that we could switch to ICGF alone seems premature. While the ICGF technique does avoid the logistic hurdles of using radioactive tracer, using ICGF would require many hospitals to buy new equipment to perform the procedure.

Most importantly, what they cannot tell us from this study is the FNR for the dual method versus ICGF alone. This is particularly concerning in a small series that included $45.9 \%$ of subjects with $\mathrm{N} 2$ or N3 disease, which some would consider unsuitable for SLNB in the first place. Conversely, in contrast to the Z1071 patients, not all of the patients had biopsy-proven nodal metastases, and the primary group also included $9 \mathrm{cN} 0$ patients, all of whom were randomized to the RI alone group. Compared with the Z1071 study, which showed that dual mapping techniques reduced the FNR to more acceptable levels, and studies showing that $20 \%$ or more of marked positive nodes are not identified as SLN by mapping, switching to a single method of mapping could lead to unacceptable FNRs. Optimizing the technique of SLN biopsy is important for any patients in whom nodal status may impact subsequent treatment, particularly for patients with positive nodes who receive NST. While simplification also is worthwhile, the primary goal should be accurate staging and optimizing therapy. Currently, it appears that best practice is to consider SLNB after NST for patients who present with $\mathrm{cN} 1$ disease and convert to cNO by imaging. SLN mapping with two dyes plus marking positive nodes with a method that facilitates detection and confirmation of their removal is the safest option. Whether ALND should be performed if a marked positive node is not found is an unsettled question, as is whether completion ALND is required if any SLN or marked nodes are still positive. The latter question is being addressed by the ongoing Alliance for Clinical Trials in Oncology A11202 trial, a randomized phase III trial evaluating the role of axillary lymph node dissection in breast cancer patients (cT1-3 N1) who have positive sentinel lymph node disease after neoadjuvant chemotherapy (NCT01901094). ${ }^{35}$

DISCLOSURES The authors declare that they have no conflict of interest.

\section{REFERENCES}

1. Giuliano AE, Kirgan DM, Guenther JM, et al. Lymphatic mapping and sentinel lymphadenectomy for breast cancer. Ann Surg. 1994;220:391-401.

2. Krag DN, Anderson SJ, Julian TB, et al. Sentinel-lymph-node resection compared with conventional axillary-lymph-node dissection in clinically node-negative patients with breast cancer: overall survival findings from the NSABP B-32 randomised phase 3 trial. Lancet Oncol. 2010;11:927-33.

3. Fisher B, Bryant J, Wolmark N, et al. Effect of preoperative chemotherapy on the outcome of women with operable breast cancer. J Clin Oncol. 1998;16:2672-85.

4. Wolmark N, Wang J, Mamounas E, et al. Preoperative chemotherapy in patients with operable breast cancer: nine-year results from National Surgical Adjuvant Breast and Bowel Project B-18. JNCI Mongraphs. 2001;30:96-102.

5. Early Breast Cancer Trial Collab Group. Long-term outcomes for neoadjuvant versus adjuvant chemotherapy in early breast cancer: meta-analysis of individual patient data from ten randomised trials. Lancet Oncol. 2018;19:27-39.

6. Ataseven B, von MG. The impact of neoadjuvant treatment on surgical options and outcomes. Ann Surg Oncol. 2016;23:3093-9.

7. Kaufmann M, von MG, Mamounas EP, et al. Recommendations from an international consensus conference on the current status 
and future of neoadjuvant systemic therapy in primary breast cancer. Ann Surg Oncol. 2012;19:1508-16.

8. Kantor O, Ajmani G, Wang C-H, et al. The shifting paradigm for breast cancer surgery in patients udergoing neoadjuvant chemotherapy. Ann Surg Oncol. 2018;25:164-72.

9. Killelea BK, Yang VQ, Mougalian S, et al. Neoadjuvant chemotherapy for breast cancer increases the rate of breast conservation: results from the National Cancer Database. J Am Coll Surg. 2015;220:1063-9.

10. Puig CA, Hoskin TL, Day CN, et al. National trends in the use of neoadjuvant chemotherapy for hormone receptor-negative breast cancer: A National Cancer Data Base Study. Ann Surg Oncol. 2017;24:1242-50.

11. Gradishar WJ, Anderson BO, et al. NCCN Guidelines Version 4.2018. Breast Cancer. 2019. National Comprehensive Cancer Network. https://www.nccn.org/professionals/physician_gls/pdf/ breast.pdf. Accessed 3 March 2019.

12. Lyman GH, Somerfield MR, Bosserman LD, et al. Sentinel lymph node biopsy for patients with early-stage breast cancer: American Society of Clinical Oncology Clinical Practice Guideline Update. J Clin Oncol. 2017;35:561-4.

13. Mamounas EP, Brown A, Anderson S, et al. Sentinel node biopsy after neoadjuvant chemotherapy in breast cancer: results from National Surgical Adjuvant Breast and Bowel Project Protocol B-27. J Clin Oncol. 2005;23:2694-702.

14. Boughey JC, Suman VJ, Mittendorf EA, et al. Sentinel lymph node surgery after neoadjuvant chemotherapy in patients with node-positive breast cancer: the ACOSOG Z1071 (Alliance) clinical trial. JAMA. 2013;310:1455-61.

15. Koolen BB, Valdes Olmos RA, Wesseling J, et al. Early assessment of axillary response with (1)(8)F-FDG PET/CT during neoadjuvant chemotherapy in stage II-III breast cancer: implications for surgical management of the axilla. Ann Surg Oncol. 2013;20:2227-35.

16. Park TS, Thomas SM, Rosenberger LH, et al. The association of extent of axillary surgery and survival in women with N2-3 invasive breast cancer. Ann Surg Oncol. 2018;25:3019-29.

17. Hieken TJ, Boughey JC, Jones KN, et al. Imaging response and residual metastatic axillary lymph node disease after neoadjuvant chemotherapy for primary breast cancer. Ann Surg Oncol. 2013;20:3199-204.

18. Rastogi P, Anderson SJ, Bear HD, et al. Preoperative chemotherapy: updates of National Surgical Adjuvant Breast and Bowel Project Protocols B-18 and B-27. J Clin Oncol. 2008;26:778-85.

19. Cortazar $\mathrm{P}$, Zhang $\mathrm{L}$, Untch $\mathrm{M}$, et al. Pathological complete response and long-term clinical benefit in breast cancer: the CTNeoBC pooled analysis. Lancet. 2014;384:164-72.

20. Masuda N, Lee SJ, Ohtani S, et al. Adjuvant capecitabine for breast cancer after preoperative chemotherapy. $N$ Engl J Med. 2017;376:2147-59.

21. von Minckwitz G, Huang CS, Mano MS, et al. Trastuzumab emtansine for residual invasive HER2-positive breast cancer. $N$ Engl J Med. 2019;380:617-28.

22. Garg AK, Buchholz TA. Influence of neoadjuvant chemotherapy on radiotherapy for breast cancer. Ann Surg Oncol. 2015;22:1434-40.

23. Fernandez LJ, Bear HD. Axillary node management in patients receiving neoadjuvant chemotherapy. Curr Breast Cancer Rep 2015;7:30-6.
24. Xing Y, Foy M, Cox DD, et al. Meta-analysis of sentinel lymph node biopsy after preoperative chemotherapy in patients with breast cancer. Br J Surg. 2006;93:539-46.

25. Kelly AM, Dwamena B, Cronin P, et al. Breast cancer sentinel node identification and classification after neoadjuvant chemotherapy-systematic review and meta-analysis. Acad Radiol. 2009;16:551-63.

26. van Deurzen CH, Vriens BE, Tjan-Heijnen VC, et al. Accuracy of sentinel node biopsy after neoadjuvant chemotherapy in breast cancer patients: a systematic review. Eur $J$ Cancer. 2009;45:3124-30.

27. Caudle AS, Yang WT, Krishnamurthy S, et al. Improved axillary evaluation following neoadjuvant therapy for patients with nodepositive breast cancer using selective evaluation of clipped nodes: implementation of targeted axillary dissection. J Clin Oncol. 2016;34:1072-8.

28. Donker M, Straver ME, Wesseling J, et al. Marking axillary lymph nodes with radioactive iodine seeds for axillary staging after neoadjuvant systemic treatment in breast cancer patients: the MARI procedure. Ann Surg. 2015;261:378-82.

29. Siso C, de TJ, Esgueva-Colmenarejo A, et al. Intraoperative ultrasound-guided excision of axillary clip in patients with nodepositive breast cancer treated with neoadjuvant therapy (ILINA Trial): a new tool to guide the excision of the clipped node after neoadjuvant treatment. Ann Surg Oncol 2018; 25:784-91.

30. Simons JM, van Nijnatten TJA, van der Pol CC, et al. Diagnostic accuracy of different surgical procedures for axillary staging after neoadjuvant systemic therapy in node-positive breast cancer: a systematic review and meta-analysis. Ann Surg. 2019;269:432-42.

31. Boughey JC, Ballman KV, Le-Petross HT, et al. Identification and resection of clipped node decreases the false-negative rate of sentinel lymph node surgery in patients presenting with nodepositive breast cancer (T0-T4, N1-N2) who receive neoadjuvant chemotherapy: results from ACOSOG Z1071 (Alliance). Ann Surg. 2016;263:802-7.

32. Choy N, Lipson J, Porter C, et al. Initial results with preoperative tattooing of biopsied axillary lymph nodes and correlation to sentinel lymph nodes in breast cancer patients. Ann Surg Oncol 2015; 22:377-382.

33. Coufal O, Zapletal O, Gabrielova L, et al. Targeted axillary dissection and sentinel lymph node biopsy in breast cancer patients after neoadjuvant chemotherapy - a retrospective study. Rozhl Chir 2018; 97:551-557.

34. Jung S-Y, Han JH, Lee E-G, et al. Comparison of sentinel lymph node biopsy using the dual method of indocyanine green fluorescence plus radioisotope versus radioisotope versus radioisotope only in breast cancer patients after neoadjuvant chemotherapy: a prospective, randomized, open-label, single center phase 2 trial. Ann Surg Oncol. 2019 (in press).

35. Alliance 11202: a randomized Phase III trial comparing axillary lymph node dissection to axillary radiation in breast cancer patients (cT1-3 N1) who have positive sentinel lymph node disease after neoadjuvant chemotherapy. 2019. Available at:https://clinicaltrials.gov/ct2/show/NCT01901094. Accessed 7 March 2019.

Publisher's Note Springer Nature remains neutral with regard to jurisdictional claims in published maps and institutional affiliations. 